\title{
OPTICAL PROPERTIES OF CARBONACEOUS MATERIALS
}

\author{
E. Bussoletti \\ Istituto Universitario Navale, Napoli (Italy) \\ L. Colangeli \\ Space Science Department of ESA, ESTEC, Noordwijk (The Netherlands)
}

\begin{abstract}
We discuss the optical properties of carbonaceous materials from the VUV to the FIR. Updated laboratory data on amorphous carbon (HAC) and some PAH mixtures are analysed and compared with relevant literature. We find that the $2175 \AA$ hump can be due to very small $(\langle a\rangle \leq 10 \AA)$ HAC grains. The UIR bands seem better explained by mixtures of HAC particles and collections of PAHs.
\end{abstract}

\section{INTRODUCTION}

During the past few years there has been a great deal of research concerning the nature of interstellar grains. Astronomical observations have spanned the VUV to the FIR, while numerous laboratory experiments have provided further knowledge on the properties of materials which are realistic candidates of interstellar dust. A contribution to this knowledge and compilation of laboratory data came from the 1987 Capri Workshop on "Experiments on Cosmic Dust Analogues" (Bussoletti, Fusco, and Longo, 1988). Thanks to the improved quality of observations which bear on the dust, and the recent availability of relevant laboratory data, we now have a much better appreciation of the diverse nature of the dust. This has prompted Mathis (1988) to summarize the situation by writing that at present we have both "an increased understanding and confusion."

Let us point out here how the situation appears. Despite their interest, many laboratory results which are prompted by astrophysical questions suffer some limitations: a) clear, detailed information about the experimental physical parameters and boundary conditions (range of applicability) is seldomly provided by the authors; $b$ ) it is quite unusual to find optical analyses for a single sample which extend over the entire wavelength range $1200 \AA-1 \mathrm{~mm}$ needed to cover astronomical observations. This situation is exasperated further as theoreticians tend to adopt different sets of experimental data in their "models", data which are obtained for materials far from having the same chemical and physical properties. In order to reduce some of these problems, the groups at Lecce and Naples have been performing a systematic analysis of different carbonaceous materials and mixtures since 1980 . 
These materials include various forms of amorphous carbons and polycyclic aromatic hydrocarbons - PAHs - expected to exist in space (Bussoletti, Colangeli, and Orofino, 1988). We will discuss here some of the most recent results, and compare them with those obtained by other authors.

\section{MATERIAL CHARACTERISTICS}

In the case of carbonaceous materials, the techniques usually adopted to produce samples are: a) laser pulses onto targets (Stephens, 1980 - hereinafter STP; and Duley, 1984), b) arc discharge between graphite or amorphous carbon electrodes (Day and Huffman, 1973; Koike, Hasegawa, and Manabe, 1980 - hereinafter KHM; Tanabe' et al., 1983; Borghesi et al., 1983), c) burning of hydrocarbons (KHM; Borghesi et al., 1983), and d) quenching of a hydrocarbon plasmic gas (Sakata et al., 1983). While process (c) is usually performed in air at ambient pressure, the other techniques are used in controlled atmospheres of inert gases at various pressures. The resulting smoke samples from a), b), and c) consist of spherical/spheroidal, submicron grains with an average radius of $40 \AA \leq<a\rangle \leq 500 \AA$. The particle size increases with pressure because of collisional growth. In two cases, however, the final product is produced as a film: 1) a poorly hydrogenated amorphous carbon (Duley, 1984), and 2) the quenched carbonaceous condensate (Sakata et al., 1983). As pointed out by Huffman (1988), when solid particles are obtained, they are so reactive that all the samples suffer the problem of clumping to some extent. This process tends to alter, sometimes severely, the optical properties expected for isolated particles. Despite the care which can be applied to disperse the samples in matrix powders, everybody has to be aware that the phenomenon cannot be avoided until new experimental methods are developed.

We have produced particles both by arc discharge from amorphous carbon electrodes - HAC (in $A r$ at 0.4 Torr) and by hydrocarbon burning - BE/XY (Benzene and Xylene in air at 760 Torr). The samples appear as fluffy clusters built up from single spheroidal grains (Borghesi, Bussoletti, and Colangeli, 1985) with log-normal size distributions and average radius respectively of $\langle a\rangle_{H A C}=40 \AA$ and $\left.<a\right\rangle_{B E}$ $=150 \AA$. Electron diffraction methods show no crystalline structure larger than a few $\AA$, so that we can call these particles amorphous or - better - "disordered". A comparison of the morphological data from all the authors previously mentioned shows a clear grain size dependence on ambient pressure: the higher the pressure, the larger the particle. However recall that the temperature may also be a critical parameter, as when $\mathrm{T}$ rises, the material evaporation rate increases favouring the collisional growth of grains.

\section{UV EXTINCTION PROPERTIES}

Particles produced by laser pulses, arc discharge, or hydrocarbon burning show a peak in the UV range which is actually weaker and broader than the interstellar bump, and which falls at wavelengths longer than $2175 \AA$. Our data agree well with those reported by KHM because of the similar production methods and laboratory parameters. The difference in the starting raw material - amorphous carbon and graphite, respectively - does not seem to affect the final results. In addition, the shape of the UV peak becomes broader and broader and the peak wavelength 
decreases in going from HAC to STP to $\mathrm{BE} / \mathrm{XY}$ samples. The hump is usually attributed to surface plasmon modes by electrons in the ground state; this identification in the previous samples is confirmed by the absence of any absorption feature at energies lower than $5.3 \mathrm{eV}$. Therefore the band changes may be interpreted as follows: a) the wider the size distribution and the larger the average size, the broader the peak, $b$ ) the larger the average size, the longer the peak wavelength. These results seem to suggest that, despite the presence of clumping problems, the optical properties of single particles are still well evident at UV wavelengths.

The film condensed by Duley (1984) presents a structure intermediate between $\mathrm{sp}^{2}$ (graphite) and $\mathrm{sp}^{3}$ (diamond) coordination, with a predominance of the latter. While this material does not show any relevant signature around $2200 \AA$, it has a rapid VUV rise. Our HAC material appears to have a similar structural composition, as suggested by its Raman spectrum (Blanco et al., 1989) and the steep rise in the VUV extinction. The different behaviour in the $2200 \AA$ region between small grains and the film may be attributed to two possible causes: a) the $\mathrm{sp}^{2} / \mathrm{sp}^{3}$ ratio in our grains is higher than in the film, or b) since the $\sim 2200 \AA$ is a typical surface resonance, films have a surface-to-volume ratio much smaller than the grained samples, so that the number of surface modes per unit area may not be sufficient to produce the feature.

\section{ASTROPHYSICAL IMPLICATIONS}

The study of the interstellar extinction curve as well as other astronomical observations have not yet provided a unique determination of the properties of the materials in the grains. Good reviews are given in the recent papers by Tielens and Allamandola (1987), Greenberg (1986), and Mathis (1988). A multicomponent grain mixture seems to be required to account for the observations. Massa and Fitzpatrick (1986) and Fitzpatrick and Massa (1986) have definitely shown that, while the $2200 \AA$ peak wavelength is fixed, the width and the strength of the UV band vary along different lines of sight. The different models of the interstellar dust developed in the past to account for the extinction curve assumed that, besides amorphous silicates, graphite had to be present in order to account for the bump (Mathis, Rumpl, and Nordsiek, 1977; Greenberg and Chlewicki, 1983; Draine and Lee, 1984). Several physical and chemical constraints tend to rule out a graphite component, at least in the regular structure which is known on the earth, as a possible major component of interstellar grains. On the contrary, it seems more reasonable that interstellar carbon consists of a regular progression from PAHs to a disordered structure which may also contain graphitic platelets.

By using our experimental results we have verified how amorphous carbon grains can match the extinction curve. As shown in $\S 3$, a decrease in $\langle a\rangle$ produces a shift of the peak to shorter wavelength and an increase of $\Gamma=A($ peak $) / A(\mathrm{~V})$. By simply extrapolating the $\mathrm{HAC}$ and $\mathrm{BE}$ extinction data we have constructed an "interstellar amorphous carbon" (IAC), with the only constraint of $\lambda_{\text {peak }}$ falling at $2175 \AA$. Bussoletti, Colangeli, and Orofino (1987) obtained an extinction curve due to grains with $\langle a\rangle=10 \AA$ which shows a good agreement with observations (Figure 1). $\Gamma_{I A C}$ is 3.8 , larger than the 3.2 value determined for diffuse clouds. In addition, only $18 \%$ of the available interstellar carbon is needed to account for the hump. The high visual extinction due to the clumping in the laboratory must be sensibly 


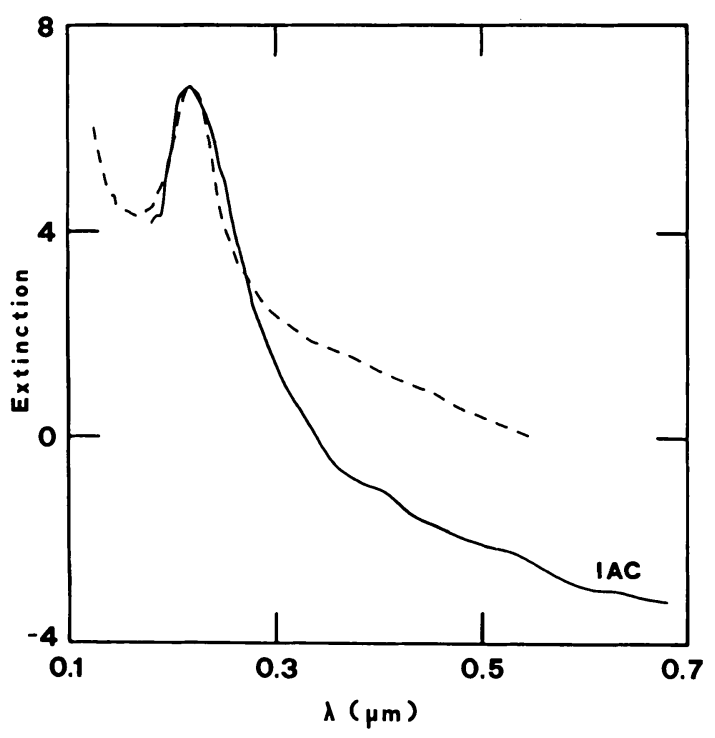

Fig. 1. Comparison between the IAC extinction and the average interstellar extinction curve (Bussoletti, Colangeli, and Orofino, 1987).

reduced in order to compare with the interstellar extinction curve. Consequently, an additional dust component, such as large polarizing amorphous silicate grains, is therefore expected to produce most of the visual extinction.

\section{INFRARED PROPERTIES}

The extinction spectra of our samples out to $300 \mu \mathrm{m}$ follow a general $\lambda^{-1.0_{-0.1}^{+0.8}}$ law. There is some dependence on the raw materials used and the wavelength range, in agreement with KHM. Tanabe' et al. (1983) found a slower trend $\propto \lambda^{-0.6}$ in the 25-250 $\mu \mathrm{m}$ range. According to Huffman (1988), isolated particles should be characterized by a steeper dependence, indicating that our samples are less clumped than those studied by Tanabe' et al.. These results indicate that most of the spectra detected in the ISM which follow a steeper law should be due to amorphous silicate rather than to carbon. However, clear evidence of a $\lambda^{-1}$ trend in the spectra of carbon stars (Jura, 1983, 1986) suggests that, at least in this case, grain emissivity is closer to that of amorphous carbon rather than that of graphite. This is confirmed by the absence of a graphite feature at $11.52 \mu \mathrm{m}$ in these objects, and means that, in the shells of $C$ stars, amorphous carbon grains form and tend to clump before the ejection into outer space. This picture is supported by the extinction measured towards R Cor Bor-type stars (Hecht et al., 1984), and towards the planetary nebula Abell 30 (Greenstein, 1981). These objects have a peak at 2400-2500 $\AA$, consistent with a distribution of particles with average dimensions between $50 \AA$ and $150 \AA$. 


\section{THE PROBLEM OF UIR BANDS}

Since their discovery, the UIR bands have represented a puzzling problem which is not yet completely understood. As most features are caused by the bending and stretching of a particular bond, rather than by a specific molecule, it appears to be still difficult to decide if they can be attributed to solid grains (Duley and Williams, 1981; Sellgren, 1984) or to PAHs (Léger and Puget, 1984; Allamandola, Tielens, and Barker, 1985). It is interesting to note that absorption spectra of our HAC samples show seven weak bands that are due to the presence of hydrogen which match the corresponding UIR band wavelengths. A comparison of HAC bands with those expected from chrysene and coronene shows that none of these three materials alone is able to match the wavelengths of all of the observed features. Some bands are common to all samples, some are present in HAC and absent in PAHs, and vice versa. Since disordered carbon grains and PAHs are formed and coexist together in the outflows of some sources, it appears reasonable to suggest that the UIR bands may be produced by a mixture of both grains and a collection of PAHs. This idea has been experimentally tested by Blanco, Bussoletti, and Colangeli (1988). Transmission spectra of a mixture of HAC grains and PAH collections have been measured; two samples of PAHs have been obtained, respectively, with a prevalent aliphatic (PAH330) and aromatic (PAH460) character. It is worithwhile to note that pure PAH spectra always appear too rich, confirming the necessity of the presence of some other material able to smear some bands as well as to produce those lacking in the PAH mixture. None of the two HAC + PAH mixtures have, however, absorption bands which correctly match all of the eleven UIR emission bands; they can account for only nine. Both mixtures lack the $8.6 \mu \mathrm{m}$ feature, while the $3.28 \mu \mathrm{m}$ feature is only marginally visible in the spectrum produced by HAC + PAH460. The 7.6 $\mu \mathrm{m}$ feature is present instead in the HAC + PAH330. These results are obviously not general and other mixtures may probably show a behaviour able to match all UIR bands. Typically, an addition of compact molecules such as methylcoronene or circobiphenyl could probably result in the appearance of an $8.6 \mu \mathrm{m}$ band. These results support, quite strongly, the idea that the overall UIR band spectrum can be better explained by a mixture composed of amorphous (disordered) carbon grains and a collection of PAHs with an intermediate character between aliphatic and aromatic.

Unfortunately, IR absorption and emission spectra of HAC and PAHs are not yet available at high temperature. Furthermore, for the molecule-sized emitters, the process is a non-equilibrium one, and temperature has no meaning. Therefore, any simulation of UIR emission bands based on temperature alone appears to be not very significant because of the large number of assumptions which are required. Blanco, Bussoletti, and Colangeli (1988) have then limited the comparison to NGC 7027, whose near IR continuum suggest a temperature of about $300 \mathrm{~K}$. Figure 2 shows the comparison between observations and the HAC+PAH460 mixture. Here we would like to stress that, within present limitations of available laboratory data, as the shape and intensity of astronomical bands depend critically upon local abundances of different carriers, we do not think it is very significant to extend further the comparison between UIR bands and those observed in laboratory. The same applies to theoretical spectra because they are based upon room temperature transmission data of single molecules, and do not seem to be able to match completely the intensity and the shape of the bands observed in space. We believe that the status of the entire subject is still at its beginning as many questions need to be answered 


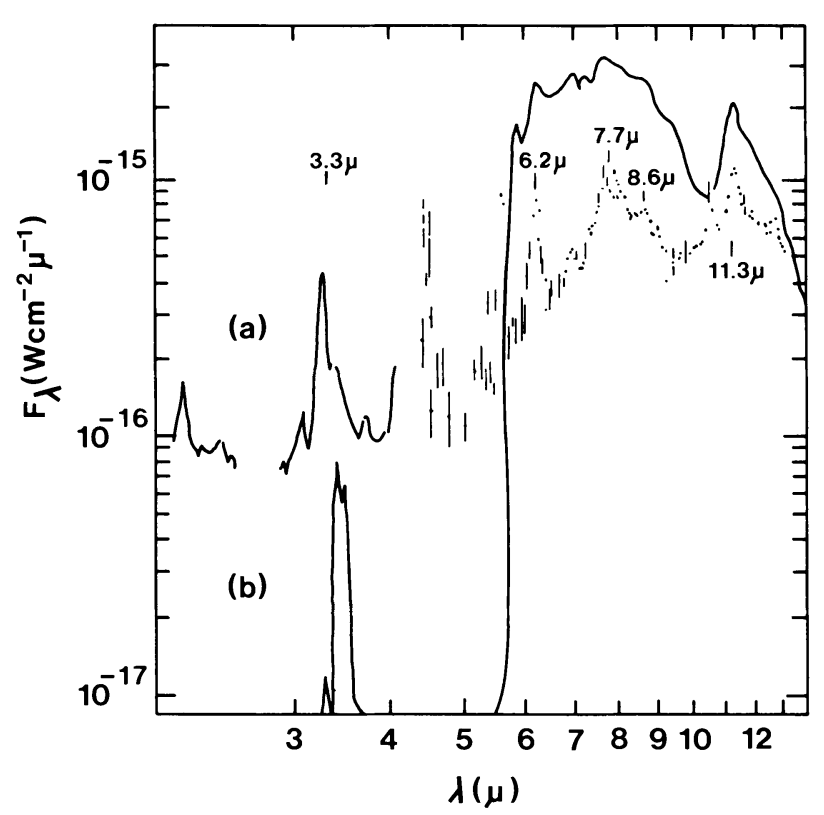

Fig. 2. (a) Comparison between the observed spectrum of NGC 7027 and (b) the emission spectrum of HAC+PAH460 for $T=300 \mathrm{~K}$ (Blanco, Bussoletti, and Colangeli, 1988).

before reaching acceptable conclusions. From an observational point of view, it is important to know the spatial behaviour of the spectra, as well as the possible existence of other features, too weak to be detected within present instrumental limitations. A substantial amount of work is also needed in the laboratory to measure the spectral characteristics at different temperatures of both neutral and ionized materials.

Finally we want to point out that a situation of uncertainty, similar to that existing for UIR bands, applies to the near IR observations of comet Halley. In the case of the well pronounced and structured emission feature at $\sim 3.36 \mu \mathrm{m}$, different kinds of gaseous molecules and/or solid grains bearing $C H-X$ bonds have been proposed as possible sources of the band (see, for instance, Knacke, Brooke, and Joyce, 1987; Moroz et al., 1987; Encrenaz et al., 1987; Colangeli et al., 1989).

ACKNOWLEDGEMENTS. This work was partially supported by contracts PSN 018.85, CNR 86.157 and CNR 87.01079.02, and by Ministero Pubblica Istruzione $60 \%$ and $40 \%$.

\section{REFERENCES}

Allamandola, L. J., Tielens, A. G. G. M., and Barker, J. R. 1985, Ap. J. (Letters), 290, L25. 
Blanco, A., Borghesi, A., Bussoletti, E., Colangeli, L., Fonti, S., Lugara, M., Orofino, V., and Scamarcio, G. 1989, in Interstellar Dust Contributed Papers, eds. A. G. G. M. Tielens and L. J. Allamandola, NASA CP-3036.

Blanco, A., Bussoletti, E., and Colangeli, L. 1988, Ap. J., 394, 875.

Borghesi, A., Bussoletti, E., Colangeli, L., Minafra, A., and Rubini, F. 1983, Infrared Phys., 23, 85.

Borghesi, A., Bussoletti, E., and Colangeli, L. 1985, Astr. Ap., 142, 225.

Bussoletti, E., Fusco, C., and Longo, G. 1988, in Experiments on Cosmic Dust Analogues, Astrophysics and Space Science Library Vol. 149, (Dordrecht: Kluwer Academic Publishers).

Bussoletti, E., Colangeli, L., and Orofino, V. 1987, Ap. J. (Letters), 821, L87.

- 1988, in Experiments on Cosmic Dust Analogues, ed. E. Bussoletti, C. Fusco, and G. Longo (Dordrecht: Kluwer Academic Publishers), p. 63.

Colangeli, L., Schwehm, G., Bussoletti, E., Blanco, A., Fonti, S., and Orofino, V. 1989, in Interstellar Dust Contributed Papers, eds. A. G. G. M. Tielens and L. J. Allamandola, NASA CP-3036.

Day K. L., and Huffman, D. R. 1973, Natire (Phys. Sci.), 243, 50.

Draine, B. T., and Lee, H. M. 1984, Ap. J., 285, 89.

Duley, W. W. 1984, Ap. J., 287, 694.

Duley, W. W., and Williams, D. A. 1981, M. N. R. A. S., 196, 269.

Encrenaz, T., Puget, J. L., Bibring, J. P., Combes, M., Crovisier, J., Emerich, C., d'Hendecourt, L., and Rocard, F. 1987, in Symposium on the Diversity and Similarity of Comets, ESA SP-278, p. 369.

Fitzpatrick, E. L., and Massa, D. 1986, Ap. J., 807, 286.

Greenberg, J. M. 1986, in Light on Dark Matter, ed. F. P. Isreal, (Dordrecht: Reidel), p. 177.

Greenberg, J. M., and Chlewicki, G. 1983, Ap. J., 272, 563.

Greenstein, J. L. 1981, Ap. J., 245, 124.

Hecht, J. H., Holm, A. V., Donn, B., and Wu, C. C. 1984, Ap. J., 280, 228.

Huffman, D. R. 1988, in Experiments on Cosmic Dust Analogues, eds. E. Bussoletti, C. Fusco, and G. Longo (Dordrecht: Kluwer Academic Publishers), p. 25.

Jura, M. 1983, Ap. J., 267, 647.

1986, Ap. J., s03, 327.

Knacke, R. F., Brooke, T. Y., and Joyce, R. R. 1987, Astr. Ap., 187, 625.

Koike C., Hasegawa, H., and Manabe, A. 1980, Ap. Space Scr., 67, 495.

Léger, A., and Puget, J. L. 1984, Astr. Ap., 137, L5.

Massa, D., and Fitzpatrick, E. L. 1986, Ap. J. Suppl., 60, 305.

Mathis, J. S. 1988, Astron. Lett. Comm., 26, 239.

Mathis, J. S., Rumpl, W., and Nordsiek, K. H. 1977, Ap. J., 217, 425.

Moroz, V. I. et al. 1987, Astr. Ap., 187, 513.

Sakata, A., Wada, S., Okutsu, Y., Shintani, H., and Nakada, Y. 1983, Nature, s01, 493.

Sellgren, K. 1984, Ap. J., $277,623$.

Stephens, J. R. 1980, Ap. J., 237, 450.

Tanabe', T., Nakada, Y., Kamijo, F., and Sakata, A. 1983, Pub. Astr. Soc. Japan, $85,397$.

Tielens, A. G. G. M., and Allamandola, L. J. 1987, in Interstellar Processes, eds. D. J. Hollenbach and H. A. Thronson, (Dordrecht: Reidel), p. 397. 


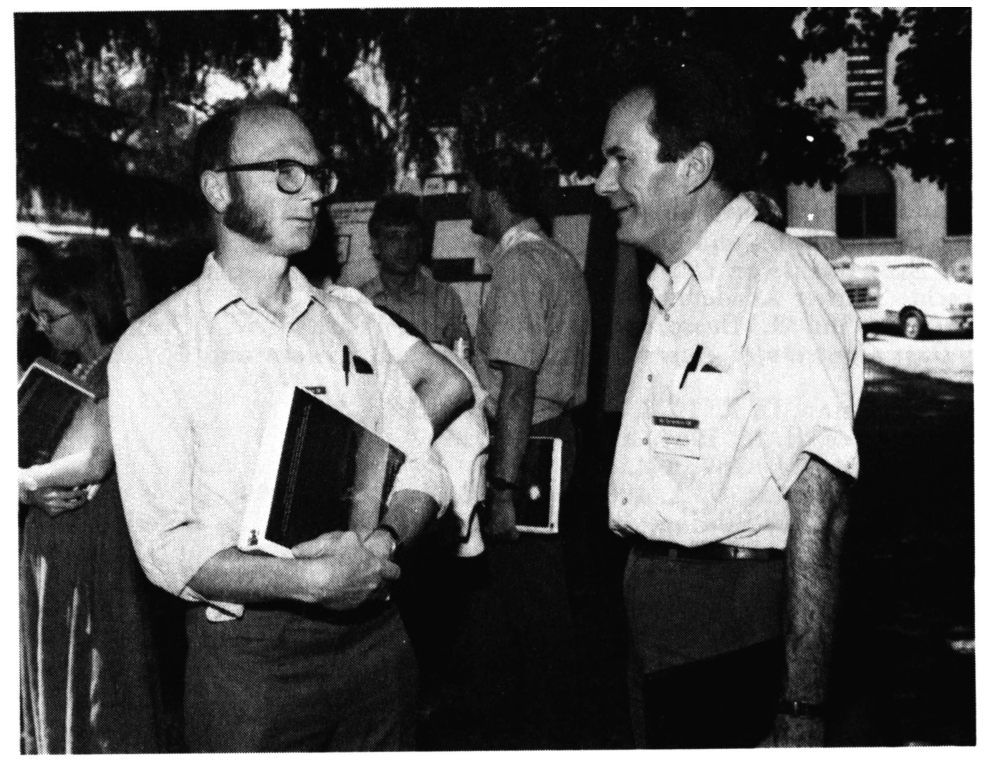

John Goebel and Jacek Krełowski 